\title{
Isolation and Biochemical Characterization of Lactobacillus species from Yogurt and Cheese samples in Dhaka Metropolitan Area
}

\author{
Sultana Juhara Mannan, Refaya Rezwan, Md. Shajidur Rahman and Kohinur Begum
}

Department of Pharmacy, ASA University Bangladesh, ASA Tower, Shyamoli, Dhaka-1207, Bangladesh.

Received: October 09, 2016; Accepted: October 31, 2016; Published (Web): March 19, 2017

\begin{abstract}
The purpose of this study was to explore Lactobacillus species from yogurt and cheese that can be used as potential probiotics. In this study, a total of twenty five samples, fifteen from cheese and ten from yogurt were collected from local markets, Dhaka city during May-July, 2016. Single colonies were isolated by enriching in MRS broth and subsequent streaking on MRS agar plate. Total twenty five isolated bacteria were identified as Lactobacillus species by morphological, gram staining and short biochemical tests. All isolated strains were characterized for probiotic properties including acid and salt tolerance, phenol tolerance, sugar fermentation, lactose fermentation and proteolytic activity. Acid tolerance test was performed at $\mathrm{pH} \mathrm{2,} \mathrm{3,} \mathrm{4,} \mathrm{5,}$ 6, 7 and 8 in MRS broth. Results showed all isolates survived in highly acidic $\mathrm{pH}$, however most of the strains also survived in alkaline media $(\mathrm{pH} 8)$. Salt tolerance test was performed at $2 \%, 4 \%$ and $8 \% \mathrm{NaCl}$ in MRS broth. All isolates survived in $2 \%$ and $4 \% \mathrm{NaCl}$ concentrations. Phenol tolerance test was performed in MRS broth with $0.1 \%, 0.2 \%, 0.3 \%$ and $0.4 \%$ phenol concentration. All strains survived in $0.1 \%$ and $0.2 \%$ phenol concentrations. Sugars such as glucose, fructose, sucrose, xylose and lactose were used for fermentation tests. Results of fermentation test showed that most isolates fermented all sugars. All strains digested casein by producing protease enzyme in skim milk agar plate. This study indicated that Lactobacillus species from yogurt and cheese samples have potential probiotic properties. Further study is needed to find specific probiotics with specific benefit from yogurt and cheese.
\end{abstract}

Key words: Lactobacillus spp., probiotic, cheese, yogurt.

\section{Introduction}

Microbes associated with various beneficial effects for humans and animals are used as probiotics. These microorganisms contribute to balance in gut flora and play important roles in maintaining health (Schrezenmeir and de Vrese, 2001). The probiotic microorganisms mainly consist of the strains of the genera Lactobacillus and Bifidobacterium, Streptococcus and some Enterococcus species (Morrow et al., 2012). These bacteria play an important role in the protection of the organism against harmful microorganisms and strengthen the host's immune system (Soccol, 2010; Pundir et al., 2013). Hutt and colleagues (2006) also reported that these bacteria inhibited enteric and urinary pathogenic bacteria. Some of these Lactobacilli strains have therapeutic properties including anti-inflammatory (Prado et al., 2008) and anti-cancer activities (Chiang et al., 2012). These bacteria are also beneficial in gastrointestinal disturbances including diarrhea, dysentery, typhoid (Tambekar and Bhutada, 2010; Cross, 2002). In Bangladesh, yogurt and cheese, locally known as dahi and ponir, respectively are produced and consumed in large amount in all parts of the country (Shahnaz et al., 2004). Many studies have reported that members of the genus Lactobacillus are widely found in dairy foods and play an important role in the manufacturing of different dairy products such as yogurt, cheese and other milk products (Keer et al., 1983; Saarela, 2002; Salminen et al., 1996; Tserovska et al., 2000, 2002). However, despite growing interest in probiotics, there is a paucity of scientific research regarding emerging uses of Lactobacillus as probiotics on local cheese and yogurts in Bangladesh. Therefore extensive studies are required for finding Lactobacillus probiotics for therapeutic benefit from these milk products. In this

Correspondence to: Prof. Dr. Kohinur Begum, E-mail: prof.kohinur@asaub.edu.bd, kohinur025@yahoo.com 
study, an attempt has been taken to find potential probiotic properties of Lactobacillus spp. from yogurt and cheese in Bangladesh.

\section{Materials and Methods}

Collection of bacterial samples: Total twenty five samples, fifteen from cheese and ten from yogurt were collected from different local markets in Dhaka during May-July, 2016. $1 \mathrm{gm}$ of sample was taken in $9 \mathrm{ml}$ of MRS Broth (Hi-Media, India) and incubated at $37^{\circ} \mathrm{C}$ for $48 \mathrm{~h}$. One loopful broth culture was streaked on MRS agar plates and incubated $48 \mathrm{hrs}$. Suspected single colonies were isolated and identified by gram staining and short biochemical tests (MacFaddin, 2000; Bergey et al., 1994). Single colony was stored in MRS agar slant for further study.

Gram staining: Gram staining test was performed for all isolated strains according to the standard procedure. A smear of single colony was prepared on a clean glass slide and the smear was allowed to air-dry and then heat fixed. The heat fixed smear was flooded with crystal violet solution and after one minute, it was washed with water and flooded with mordant Gram's iodine. The smear was decolorized with $95 \%$ ethyl alcohol and rinsed with water. Finally safranin was used as counter stains for $60-80 \mathrm{sec}$ and washed with water, and examined under oil immersion (100X). Staphylococcus aureus ATCC 25923 and Escherichia coli ATCC 25922 were used as positive and negative control, respectively.

Catalase test: A drop of 3\% hydrogen peroxide was added to a fresh culture on a sterile glass slide and mixed well. Producing bubble or froth, indicated catalase-positive and no bubble or froth indicated catalase negative. Staphylococcus aureus ATCC 25923 and E. coli ATCC 25922 were used as positive and negative control, respectively.

Kliger's Iron Agar (KIA) test: All isolates were tested for KIA test to know the mode of glucose and lactose utilization. Fresh culture was inoculated by stabbing the butt and streaking the slant. After incubation at $37^{\circ} \mathrm{C}$ for $24 \mathrm{~h}$, results were recorded for color changes of the butt or slant, $\mathrm{H}_{2} \mathrm{~S}$ or other gas production. The results were observed as alkaline slant and acid butt for fermentation of glucose only, acid slant and alkaline butt for fermentation of lactose only, acid in both slant and butt for fermentation of both lactose and glucose whereas alkaline in both slant and butt for fermentation of neither lactose nor glucose. Production of hydrogen sulphide made blacking of the medium and the gas production give rise to bubble formation in the tube. S. aureus ATCC 25923 was used as positive control.

pH tolerance test: MRS broth at $\mathrm{pH} 2,3,4,5,6,7$ and 8 were prepared by adjusted with $10 \mathrm{~N} \mathrm{HCl}$ and $1 \mathrm{~N}$ $\mathrm{NaOH}$. Fresh bacterial cultures were inoculated into respective MRS broth in test tubes and incubated at $37^{\circ} \mathrm{C}$ for $48 \mathrm{~h}$. Only media was used as negative control. Results were obtained by observing turbidity of the culture media after $24 \mathrm{~h}$ and $48 \mathrm{~h}$ and no growth was observed in negative control.

$\mathrm{NaCl}$ tolerance test: $\mathrm{NaCl}$ tolerance of isolated Lactobacillus was determined by using MRS broth with $2 \%, 4 \%$ and $8 \%$ of $\mathrm{NaCl}$ concentration. Fresh culture was inoculated and incubated at $37^{\circ} \mathrm{C}$ for $48 \mathrm{~h}$. Only media was used as negative control. Results were determined by observing the turbidity after $24 \mathrm{~h}$ and 48 $\mathrm{h}$ and no growth was observed in negative control.

Phenol tolerance test: MRS broth containing $0.1 \%$, $0.2 \%, 0.3 \%$ and $0.4 \%$ of phenol concentration were prepared for the determination of phenol tolerance. Fresh culture was inoculated and incubated at $37^{\circ} \mathrm{C}$ for $48 \mathrm{~h}$. Only media was used as negative control. Results were determined by observing turbidity after $24 \mathrm{~h}$ and $48 \mathrm{~h}$ and no growth was found in negative control.

Determination of sugar fermentation: Sugar fermentation test was performed using $1 \%(\mathrm{w} / \mathrm{v})$ sugar in MRS broth. Glucose, fructose, sucrose, xylose and lactose were used in this test. Phenol red solution was used as indicator. $10 \mathrm{ml}$ media was dispensed and Durham's tube was inserted invertably in each of test tubes. Fresh culture was inoculated and incubated at $37^{\circ} \mathrm{C}$ for $24 \mathrm{~h}$. Only media was used as negative control. Results were observed by color changing and gas formation.

Casein digestion test: The protease activity was performed using MRS agar plate containing $1 \%$ skim milk solution. Bacterial cultures were inoculated and incubated for $48 \mathrm{~h}$ at $37^{\circ} \mathrm{C}$. Clear zones around the cultures indicated protease activity (Smibert and Krieg, 
1994). Pseudomonas spp. and Klebsiella spp. were used as positive and negative control, respectively.

\section{Results and Discussion}

Lactobacilli species from fermented foods are an important source of probiotics. Yogurt is one of the best known foods that contain probiotics (Oskar et al., 2004). Therefore, focus of the present study was to find Lactobacillus strains from local fermented foods yogurt and cheese in Dhaka. In this experiment, total 25 Lactobacillus spp. were identified on the basis of characteristic morphology, catalase negative and gram positive rod shape (Table $1 \& 2$ and Figure $1 \& 2$ ). Previously, Lactobacillus spp. were identified and confirmed by gram positive and catalase negative results (Rao et al., 2015; Salvetti et al., 2012; Vyas et al., 2014). However, another report showed that Lactobacilli are genetically and physiologically diverse

Table 1. Colony morphology of isolated bacteria.

\begin{tabular}{lll}
\hline Isolate no. & Morphology of colony & $\begin{array}{c}\text { Color of } \\
\text { colony }\end{array}$ \\
\hline KL1 & Small, circular, regular & Creamy \\
KL2 & Small, circular, regular & Creamy \\
KL3 & Large, circular, irregular & Creamy \\
KL4 & Small, circular, irregular & Creamy \\
KL5 & Small, circular, irregular & Creamy \\
KL6 & Small, circular, regular & Creamy \\
KL7 & Small, circular, regular & Creamy \\
KL8 & Small, circular, regular & Creamy \\
KL9 & Small, circular, regular & Creamy \\
KL10 & Small, circular, regular & Creamy \\
KL11 & Small, circular, regular & Creamy \\
KL12 & Small, circular, regular & Creamy \\
KL13 & Large, circular, regular & Creamy \\
KL14 & Small, circular, regular & Creamy \\
KL15 & Small, circular, regular & Creamy \\
KL16 & Large, circular, regular & Creamy \\
KL17 & Small, circular, regular & Creamy \\
KL18 & Small, circular, regular & Creamy \\
KL19 & Small, circular, regular & Creamy \\
KL20 & Small, circular, regular & Creamy \\
KL21 & Small, circular, regular & Creamy \\
KL22 & Small, circular, regular & Creamy \\
KL23 & Large, circular, regular & Creamy \\
KL24 & Small, circular, irregular & Creamy \\
KL25 & Small, circular, regular & Creamy \\
\hline & &
\end{tabular}

Table 2. Results of biochemical tests of isolated bacteria.

\begin{tabular}{|c|c|c|c|}
\hline $\begin{array}{l}\text { Isolate } \\
\text { no. }\end{array}$ & $\begin{array}{l}\text { Gram } \\
\text { staining }\end{array}$ & Catalase & KIA \\
\hline KL1 & Bacilli & - & Alkaline/acid \\
\hline KL2 & Bacilli & - & Alkaline/alkaline \\
\hline KL3 & Bacilli & - & Alkaline/alkaline \\
\hline KL4 & Bacilli & - & Alkaline/alkaline \\
\hline KL5 & Bacilli & - & Acid/acid \\
\hline KL6 & Coccobacilli & - & Alkaline/alkaline \\
\hline KL7 & Coccobacilli & - & Alkaline/alkaline \\
\hline KL8 & Coccobacilli & - & Acid/acid \\
\hline KL9 & Coccobacilli & - & Acid/acid \\
\hline KL10 & Coccobacilli & - & Acid/acid \\
\hline KL11 & Bacilli & - & Alkaline/alkaline \\
\hline KL12 & Bacilli & - & Alkaline/alkaline \\
\hline KL13 & Bacilli & - & Alkaline/alkaline \\
\hline KL14 & Bacilli & - & Acid/acid \\
\hline KL15 & Bacilli & - & Alkaline/alkaline \\
\hline KL16 & Bacilli & - & Alkaline/alkaline \\
\hline KL17 & Bacilli & - & Acid/alkaline \\
\hline KL18 & Bacilli & - & Acid/acid \\
\hline KL19 & Bacilli & - & Acid/acid \\
\hline KL20 & Bacilli & - & Alkaline/alkaline \\
\hline KL21 & Coccobacilli & - & Acid/acid \\
\hline KL22 & Coccobacilli & - & Acid/acid \\
\hline KL23 & Coccobacilli & - & Alkaline /alkaline \\
\hline KL24 & Coccobacilli & - & Alkaline /alkaline \\
\hline KL25 & Coccobacilli & - & Acid/acid \\
\hline
\end{tabular}

$+=$ positive; $-=$ negative; KIA: Slant/Butt

group of rod-shaped, gram-positive, catalase negative bacteria (Hoque et al., 2010). Although many studies have reported Lactobacillus strains found in human gut including oral cavity. In this study, survivability study in different $\mathrm{pH}$ was performed because $\mathrm{pH}$ is an important factor for the growth of bacteria. All strains were tested in both acidic and alkaline conditions at different ranges of $\mathrm{pH} 2$ to 8 . From this experiment, isolated Lactobacillus showed maximum growth at $\mathrm{pH}$ 2. Therefore, these bacteria exhibited survival in both highly acidic and moderate alkaline conditions and less growth in relatively neutral pH 6 and 7 (Table 3). In previous study, Pundir R.K. et al. (2013) reported that Lactobacillus isolated from fresh vegetable, fruits, curds survived in $\mathrm{pH} 3.5$ to $\mathrm{pH}$ 7.0. Another study by Hoque M.Z. et al. (2010) found Lactobacillus growth in $\mathrm{pH} 2.5$ to $\mathrm{pH}$ 8.5. The reason for choosing this $\mathrm{pH}$ range was to determine whether the isolated strains can grow in both acidic and alkaline conditions. $\mathrm{pH}$ 
tolerance is essential criteria to grow and perform their beneficial effect in the gastrointestinal tract but range of tolerance is not specified. Another test was performed to evaluate $\mathrm{NaCl}$ tolerance of Lactobacillus spp. in this study. $\mathrm{NaCl}$ is an inhibitory substance that inhibited the growth of some bacteria (Hoque et al., 2010). $\mathrm{NaCl}$ tolerance test was performed at $2 \%, 4 \%$ and $8 \% \mathrm{NaCl}$ concentrations. All the isolates were able to grow at $2 \%$ and $4 \% \mathrm{NaCl}$ concentration but did not grow at $8 \%$ $\mathrm{NaCl}$ (Table 3). This is in agreement with Forhad et al. (2015) that Lactobacillus species were not able to grow in high $\mathrm{NaCl}$ concentrations. Phenol is also an inhibitory compound produced in deamination reaction of amino acids in intestine (Suskovic et al., 1997). Probiotic bacterial strains survive low concentrations of phenol. In the present study, phenol tolerance test was performed in $0.1 \%, 0.2 \%, 0.3 \%$ and $0.4 \%$ of phenol concentration. All the isolates survived at $0.1 \%$ and $0.2 \%$ concentration while $50 \%$ isolates survived in $0.3 \%-0.4 \%$ phenol concentrations. A similar report was published by Hoque (2010), where most Lactobacillus strains survived up to $0.3 \%$ phenol.

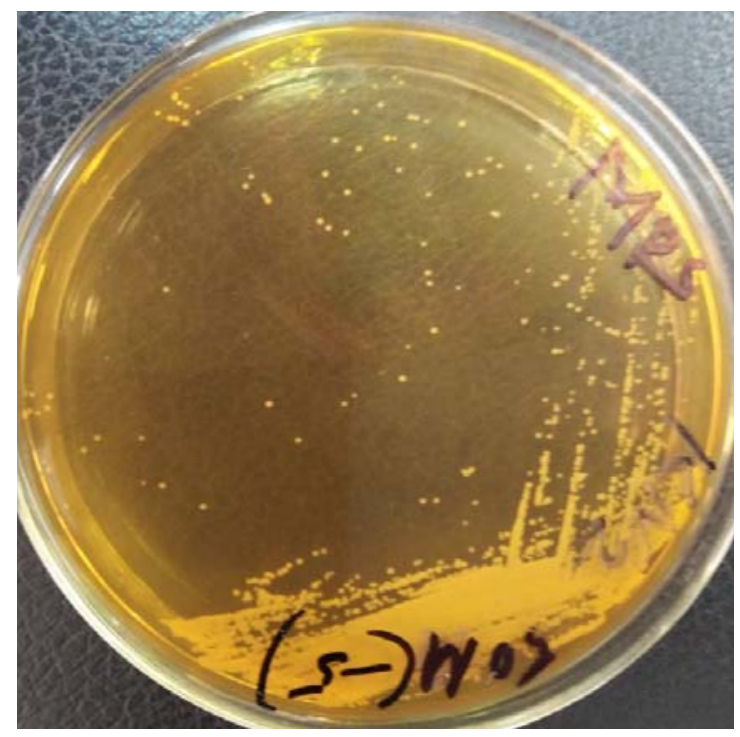

Figure 1. Lactobacillus species in MRS plate.

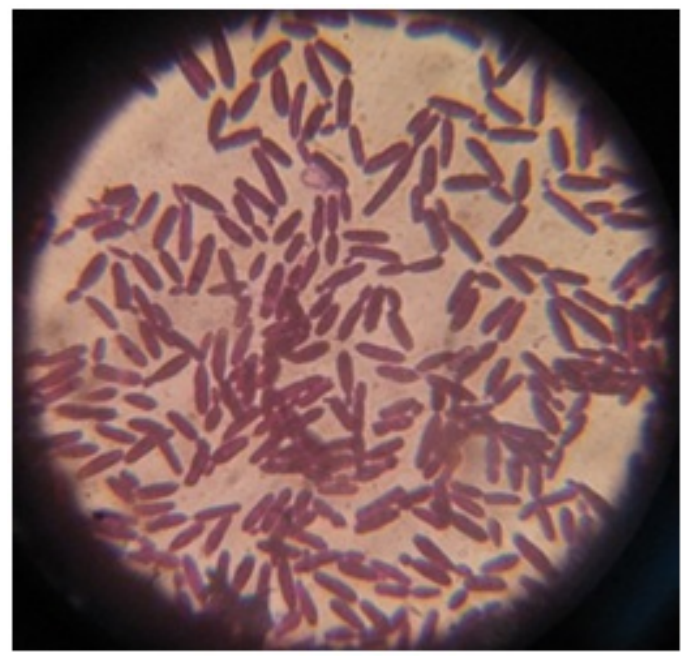

(i) Bacilli

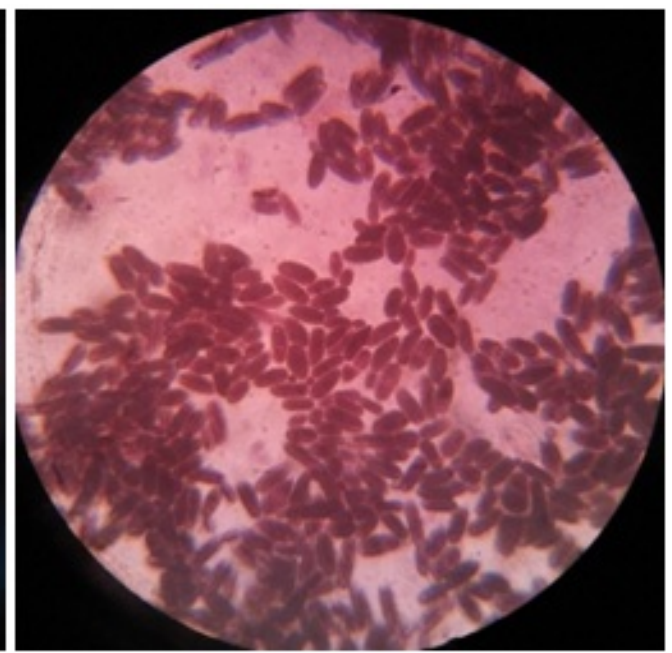

(ii) Coccobacilli

Figure 2. Gram positive Lactobacilli species in gram staining test. 
Table 3. Results of $\mathrm{NaCl}$ and pH tolerance tests of isolated Lacobacillus species.

\begin{tabular}{|c|c|c|c|c|c|c|c|c|c|c|c|c|c|c|c|c|c|}
\hline \multirow[b]{2}{*}{ Isolate } & \multicolumn{2}{|l|}{$\mathrm{pH} 2$} & \multicolumn{2}{|l|}{$\mathrm{pH} 3$} & \multicolumn{2}{|l|}{$\mathrm{pH} 4$} & \multicolumn{2}{|l|}{$\mathrm{pH} 5$} & \multicolumn{2}{|l|}{$\mathrm{pH} 6$} & \multicolumn{2}{|l|}{$\mathrm{pH} 7$} & \multicolumn{2}{|l|}{$\mathrm{pH} 8$} & \multicolumn{3}{|c|}{$\mathrm{NaCl}$ concentration } \\
\hline & $24 \mathrm{~h}$ & $48 \mathrm{~h}$ & $24 \mathrm{~h}$ & $48 \mathrm{~h}$ & $24 \mathrm{~h}$ & $48 \mathrm{~h}$ & $24 \mathrm{~h}$ & $48 \mathrm{~h}$ & $24 \mathrm{~h}$ & $48 \mathrm{~h}$ & $24 \mathrm{~h}$ & $48 \mathrm{~h}$ & $24 \mathrm{~h}$ & $48 \mathrm{~h}$ & $2 \%$ & $4 \%$ & $8 \%$ \\
\hline रd. 1 & +++ & +++ & ++ & ++ & + & + & + & ++ & - & + & + & + & ++ & ++ & +++ & + & - \\
\hline KL2 & +++ & +++ & ++ & ++ & + & ++ & + & ++ & - & + & - & + & ++ & ++ & +++ & +++ & $+/-$ \\
\hline KL3 & +++ & +++ & ++ & ++ & + & + & + & ++ & - & + & - & + & ++ & ++ & + & +++ & - \\
\hline KL4 & +++ & +++ & ++ & ++ & + & + & + & ++ & - & + & - & - & ++ & ++ & +++ & +++ & $+/-$ \\
\hline KL5 & +++ & +++ & ++ & ++ & + & ++ & + & ++ & - & - & - & $+/-$ & ++ & ++ & +++ & ++ & - \\
\hline KL6 & +++ & +++ & ++ & ++ & + & ++ & + & ++ & - & + & - & + & ++ & ++ & ++ & + & - \\
\hline KL 7 & +++ & +++ & ++ & ++ & + & + & ++ & ++ & - & + & - & + & ++ & ++ & +++ & + & $+/-$ \\
\hline KL8 & +++ & +++ & ++ & ++ & + & ++ & ++ & ++ & - & + & - & + & ++ & ++ & +++ & ++ & - \\
\hline KL9 & +++ & +++ & ++ & ++ & + & + & ++ & ++ & - & + & - & + & ++ & ++ & +++ & ++ & $+/-$ \\
\hline KL10 & +++ & +++ & ++ & ++ & + & ++ & ++ & ++ & - & + & - & + & ++ & ++ & + & ++ & - \\
\hline KL11 & $+/-$ & +++ & + & ++ & + & ++ & + & + & - & + & + & + & ++ & ++ & ++ & +++ & + \\
\hline KL12 & $+/-$ & +++ & ++ & ++ & + & - & + & + & - & + & $+/-$ & + & ++ & ++ & ++ & +++ & - \\
\hline KL13 & +++ & +++ & + & ++ & + & ++ & + & ++ & - & + & $+/-$ & - & ++ & ++ & +++ & ++ & - \\
\hline KL15 & $+/-$ & +++ & + & ++ & + & $+/-$ & + & ++ & - & + & $+/-$ & + & ++ & ++ & +++ & ++ & - \\
\hline KL16 & +++ & +++ & ++ & ++ & $+/-$ & + & + & ++ & - & + & $+/-$ & - & ++ & ++ & +++ & +++ & $+/-$ \\
\hline KL17 & +++ & +++ & ++ & ++ & $+/-$ & + & ++ & ++ & - & + & $+/-$ & - & ++ & ++ & +++ & +++ & - \\
\hline KL18 & +++ & +++ & ++ & ++ & + & + & + & + & - & + & $+/-$ & - & ++ & ++ & +++ & $+/-$ & - \\
\hline KL19 & +++ & +++ & ++ & ++ & + & + & + & ++ & - & + & $+/-$ & - & ++ & ++ & +++ & ++ & - \\
\hline KL20 & +++ & +++ & ++ & ++ & + & + & + & + & - & + & + & + & ++ & ++ & +++ & ++ & - \\
\hline KL21 & +++ & +++ & ++ & ++ & + & $+/-$ & + & + & - & + & + & + & ++ & ++ & ++ & + & $+/-$ \\
\hline KL22 & +++ & +++ & ++ & ++ & + & + & + & + & - & + & + & + & - & - & + & +++ & - \\
\hline KL23 & +++ & +++ & ++ & ++ & + & + & ++ & ++ & - & + & + & + & ++ & ++ & ++ & + & $+/-$ \\
\hline KL24 & +++ & +++ & ++ & ++ & + & $+/-$ & + & + & - & + & + & + & ++ & ++ & +++ & $+/-$ & $+/-$ \\
\hline KL25 & +++ & +++ & + & ++ & + & $+/-$ & ++ & + & - & + & + & + & ++ & ++ & + & ++ & - \\
\hline
\end{tabular}

- = no growth (negative) $+=$ slight growth (positive) $;++=$ moderate growth (positive);

$+++=$ dense growth (positive); $+/-=$ ambiguous/undecided

Table 4. Results of sugar fermentation and casein digestion tests of Lacobacillus species.

\begin{tabular}{|c|c|c|c|c|c|c|}
\hline Isolate no. & Glucose & Fructose & Sucrose & Xylose & Lactose & Casein digestion \\
\hline KL1 & $\mathrm{A}^{+}, \mathrm{G}^{+}$ & $\mathrm{A}^{+}, \mathrm{G}^{+}$ & $\mathrm{A}^{+}, \mathrm{G}^{+}$ & $\mathrm{A}^{+}, \mathrm{G}^{-}$ & $\mathrm{A}^{+}, \mathrm{G}^{+}$ & + \\
\hline KL2 & $\mathrm{A}^{+}, \mathrm{G}^{+}$ & $\mathrm{A}^{+}, \mathrm{G}^{+}$ & $\mathrm{A}^{+}, \mathrm{G}^{+}$ & $\mathrm{A}^{+}, \mathrm{G}^{+}$ & $\mathrm{A}^{+}, \mathrm{G}^{+}$ & + \\
\hline KL3 & $\mathrm{A}^{+}, \mathrm{G}^{+}$ & $\mathrm{A}^{+}, \mathrm{G}^{+}$ & $\mathrm{A}^{+}, \mathrm{G}^{+}$ & $\mathrm{A}^{+}, \mathrm{G}^{+}$ & $\mathrm{A}^{+}, \mathrm{G}^{+}$ & + \\
\hline KL4 & $\mathrm{A}^{+}, \mathrm{G}^{+}$ & $\mathrm{A}^{+}, \mathrm{G}^{+}$ & $\mathrm{A}^{+}, \mathrm{G}^{+}$ & $\mathrm{A}^{+}, \mathrm{G}^{+}$ & $\mathrm{A}^{+}, \mathrm{G}^{+}$ & + \\
\hline KL5 & $\mathrm{A}^{+}, \mathrm{G}^{+}$ & $\mathrm{A}^{-}, \mathrm{G}^{-}$ & $\mathrm{A}^{-}, \mathrm{G}^{-}$ & $\mathrm{A}^{+}, \mathrm{G}^{+}$ & $\mathrm{A}^{+}, \mathrm{G}^{+}$ & + \\
\hline KL6 & $\mathrm{A}^{+}, \mathrm{G}^{+}$ & $\mathrm{A}^{+}, \mathrm{G}^{+}$ & $\mathrm{A}^{+}, \mathrm{G}^{+}$ & $\mathrm{A}^{+}, \mathrm{G}^{-}$ & $\mathrm{A}^{+}, \mathrm{G}^{+}$ & + \\
\hline KL7 & $\mathrm{A}^{+}, \mathrm{G}^{+}$ & $\mathrm{A}^{-}, \mathrm{G}^{-}$ & $\mathrm{A}^{-}, \mathrm{G}^{-}$ & $\mathrm{A}^{+}, \mathrm{G}^{+}$ & $\mathrm{A}^{+}, \mathrm{G}^{+}$ & + \\
\hline KL8 & $\mathrm{A}^{+}, \mathrm{G}^{+}$ & $\mathrm{A}^{+}, \mathrm{G}^{+}$ & $\mathrm{A}^{+}, \mathrm{G}^{+}$ & $\mathrm{A}^{+}, \mathrm{G}^{+}$ & $\mathrm{A}^{+}, \mathrm{G}^{+}$ & + \\
\hline KL9 & $\mathrm{A}^{+}, \mathrm{G}^{+}$ & $\mathrm{A}^{+}, \mathrm{G}^{+}$ & $\mathrm{A}^{+}, \mathrm{G}^{+}$ & $\mathrm{A}^{+}, \mathrm{G}^{+}$ & $\mathrm{A}^{+}, \mathrm{G}^{+}$ & + \\
\hline KL10 & $\mathrm{A}^{+}, \mathrm{G}^{+}$ & $\mathrm{A}^{+}, \mathrm{G}^{+}$ & $\mathrm{A}^{+}, \mathrm{G}^{+}$ & $\mathrm{A}^{+}, \mathrm{G}^{+}$ & $\mathrm{A}^{+}, \mathrm{G}^{+}$ & + \\
\hline KL11 & $\mathrm{A}^{+}, \mathrm{G}^{+}$ & $\mathrm{A}^{-}, \mathrm{G}^{-}$ & $\mathrm{A}^{-}, \mathrm{G}^{-}$ & $\mathrm{A}^{+}, \mathrm{G}^{+}$ & $\mathrm{A}^{+}, \mathrm{G}^{+}$ & + \\
\hline KL12 & $\mathrm{A}^{+}, \mathrm{G}^{+}$ & $\mathrm{A}^{-}, \mathrm{G}^{-}$ & $\mathrm{A}^{-}, \mathrm{G}^{-}$ & $\mathrm{A}^{-}, \mathrm{G}^{-}$ & $\mathrm{A}^{+}, \mathrm{G}^{+}$ & + \\
\hline KL13 & $\mathrm{A}^{+}, \mathrm{G}^{+}$ & $\mathrm{A}^{+}, \mathrm{G}^{+}$ & $\mathrm{A}^{+}, \mathrm{G}^{+}$ & $\mathrm{A}^{+}, \mathrm{G}^{+}$ & $\mathrm{A}^{+}, \mathrm{G}^{+}$ & + \\
\hline KL14 & $\mathrm{A}^{+}, \mathrm{G}^{+}$ & $\mathrm{A}^{+}, \mathrm{G}^{+}$ & $\mathrm{A}^{+}, \mathrm{G}^{+}$ & $\mathrm{A}^{+}, \mathrm{G}^{+}$ & $\mathrm{A}^{+}, \mathrm{G}^{+}$ & + \\
\hline KL15 & $\mathrm{A}^{+}, \mathrm{G}^{+}$ & $\mathrm{A}^{+}, \mathrm{G}^{+}$ & $\mathrm{A}^{+}, \mathrm{G}^{+}$ & $\mathrm{A}^{+}, \mathrm{G}^{+}$ & $\mathrm{A}^{+}, \mathrm{G}^{+}$ & + \\
\hline KL16 & $\mathrm{A}^{+}, \mathrm{G}^{+}$ & $\mathrm{A}^{+}, \mathrm{G}^{+}$ & $\mathrm{A}^{+}, \mathrm{G}^{+}$ & $\mathrm{A}^{+}, \mathrm{G}^{-}$ & $\mathrm{A}^{+}, \mathrm{G}^{-}$ & + \\
\hline KL17 & $\mathrm{A}^{+}, \mathrm{G}^{+}$ & $\mathrm{A}^{+}, \mathrm{G}^{+}$ & $\mathrm{A}^{+}, \mathrm{G}^{+}$ & $\mathrm{A}^{+}, \mathrm{G}^{+}$ & $\mathrm{A}^{+}, \mathrm{G}^{+}$ & + \\
\hline KL18 & $\mathrm{A}^{+}, \mathrm{G}^{+}$ & $\mathrm{A}^{+}, \mathrm{G}^{+}$ & $\mathrm{A}^{+}, \mathrm{G}^{+}$ & $\mathrm{A}^{+}, \mathrm{G}^{-}$ & $\mathrm{A}^{+}, \mathrm{G}^{+}$ & + \\
\hline KL19 & $\mathrm{A}^{+}, \mathrm{G}^{+}$ & $\mathrm{A}^{+}, \mathrm{G}^{+}$ & $\mathrm{A}^{+}, \mathrm{G}^{+}$ & $\mathrm{A}^{+}, \mathrm{G}^{-}$ & $\mathrm{A}^{+}, \mathrm{G}^{+}$ & + \\
\hline KL20 & $\mathrm{A}^{+}, \mathrm{G}^{+}$ & $\mathrm{A}^{+}, \mathrm{G}^{+}$ & $\mathrm{A}^{+}, \mathrm{G}^{+}$ & $\mathrm{A}^{+}, \mathrm{G}^{+}$ & $\mathrm{A}^{+}, \mathrm{G}^{+}$ & + \\
\hline KL21 & $\mathrm{A}^{+}, \mathrm{G}^{+}$ & $\mathrm{A}^{+}, \mathrm{G}^{+}$ & $\mathrm{A}^{-}, \mathrm{G}^{-}$ & $\mathrm{A}^{+}, \mathrm{G}^{-}$ & $\mathrm{A}^{+}, \mathrm{G}^{+}$ & + \\
\hline KL22 & $\mathrm{A}^{+}, \mathrm{G}^{+}$ & $\mathrm{A}^{+}, \mathrm{G}^{+}$ & $\mathrm{A}^{+}, \mathrm{G}^{+}$ & $\mathrm{A}^{+}, \mathrm{G}^{-}$ & $\mathrm{A}^{+}, \mathrm{G}^{+}$ & + \\
\hline KL23 & $\mathrm{A}^{+}, \mathrm{G}^{+}$ & $\mathrm{A}^{+}, \mathrm{G}^{+}$ & $\mathrm{A}^{-}, \mathrm{G}^{-}$ & $\mathrm{A}^{+}, \mathrm{G}^{+}$ & $\mathrm{A}^{+}, \mathrm{G}^{-}$ & + \\
\hline KL24 & $\mathrm{A}^{+}, \mathrm{G}^{+}$ & $\mathrm{A}^{-}, \mathrm{G}^{-}$ & $\mathrm{A}^{+}, \mathrm{G}^{+}$ & $\mathrm{A}^{-}, \mathrm{G}^{-}$ & $\mathrm{A}^{+}, \mathrm{G}^{+}$ & + \\
\hline KL25 & $\mathrm{A}^{+}, \mathrm{G}^{+}$ & $\mathrm{A}^{+}, \mathrm{G}^{+}$ & $\mathrm{A}^{+}, \mathrm{G}^{+}$ & $\mathrm{A}^{+}, \mathrm{G}^{-}$ & $\mathrm{A}^{+}, \mathrm{G}^{+}$ & + \\
\hline
\end{tabular}

$\mathrm{A}^{+}=$Positive Acid; $\mathrm{A}^{-}=$Negative Acid; $\mathrm{G}^{+}=$Positive Gas; $\mathrm{G}^{-}=$Negative Gas; + = positive 
Lactobacillus species is a well known food fermenter worldwide. Lactobacillus bacteria fermented different monosaccharide and disaccharide (Pyar and Peh, 2014). In this study, the results of sugar fermentation were shown in table 4. All the isolates fermented glucose and lactose with evolution of gas. $92 \%, 80 \%$ and $76 \%$ of isolates fermented xylose, fructose and sucrose, respectively. The probiotic bacteria were capable of fermenting different sugars and end product is lactic acid (Klaenhammer and de Vos, 2011). This is especially useful for lactose intolerant people who cannot metabolize lactose due to lack of $\beta$-galactosidase enzyme. Therefore, fermented foods under study can help lactose intolerant persons to consume milk or lactose containing products. Lactose and dextrose utilization by Lactobacillus was confirmed by lactose and glucose utilization test. Lactobacillus digests casein in order to grow in milk and subsequently utilize the degradation products (Hayes et al., 2007). In the present study, all the isolated strains were found to digest casein indicating Lactobacillus produced protease enzyme. Atanasova et al. (2014) reported in their study that, Lactobacilli species utilized casein by proteolytic activity.

\section{Conclusion}

In this study, Lactobacillus species were isolated and identified from local cheese and yogurt and they can survive in extreme acidic and moderate alkaline conditions as well as in high concentrations of salt and low concentrations of phenol. These findings revealed that the isolated Lactobacillus strains are suitable to survive in the environment of human gastrointestinal tract. However, the strains also have probiotic potential for sugar fermentation, lactose intolerance and casein digestion. Further studies are needed in order to find specific probiotics with specific benefit from yogurt and cheese.

\section{References}

Atanasova, J., Moncheva, P. and Ivanova, I. 2014. Proteolytic and antimicrobial activity of lactic acid bacteria grown in goat milk. Biotechnol Biotechnol Equip. 28, 1073-1078.
Bergey, D.H., Holt, J.G., Krieg, N.R. and Sneath, P.H.A. 1994. Bergey's Manual of Determinative Bacteriology $\left(9^{\text {th }}\right.$ ed.). Lippincott Williams and Wilkins. Brown (2012). Bensons Microbiological applications; laboratory manual in general Microbiology, short version, $12^{\text {th }}$ ed. McGraw-Hill, NY.

Chiang, S.S. and Pan, T.M. Beneficial effects of Lactobacillus paracasei subsp. paracasei NTU 101 and its fermented products. Appl. Microbiol. Biotechnol. 93, 903-916.

Cross, M.L. 2002. Microbes versus microbes: immune signals generated by probiotic Lactobacilli and their role in protection against microbial pathogens. FEMS Immunol. Med. Microbiol. 34, 245-253.

Hayes, M., Stanton, C., Slattery, H., O'Sullivan, O., Hill, C., Fitzgerald, G. F. and Ross, R. P. 2007. Casein fermentate of Lactobacillus animalis DPC6134 contains a range of novel propeptide angiotensin-converting enzyme inhibitors. Appl. Environ. Microbiol. 73, 4658-4667. DOI 10.1128/AEM.00096-07

Hoque, M.Z., Akter, F., Hossain, K.M., Rahman, M.S.M., Billah, M.M. and Islam, K.M.D. 2010. Isolation, identification and analysis of probiotic properties of Lactobacillus spp. from selective regional yoghurts. World J. Dairy Food Sci. 5, 39-46.

Hutt, P., Shchepetova, J., Loivukene, K., Kullisaar, T. and Mikelsaar, M., 2006. Antagonistic activity of probiotic Lactobacilli and Bifidobacteria against entero and uropathogens. J. Appl. Microbiol. 100, 1324-1332.

Keer, C., Melkebeke, L., Vertriest, W., Hoozee, G. and Schoonenberghe, E. 1983. Growth of Lactobacillus species on different media. J. Inst. Brew. 89, 361-363.

Klaenhammer T.R. and de Vos W.M. 2011. An incredible scientific journey. The evolutionary tale of the lactic acid bacteria. In: Ledeboer A, Hugenholtz J, Kok J, Konings W, Wouters J (ed.) The 10th LAB symposium. Thirty years of research on lactic acid bacteria. 24 Media Labs, pp. 1-11.

MacFaddin, J.F. 2000. Biochemical Tests for Identification of Medical Bacteria. $3^{\text {rd }}$ ed. Philadelphia: Lippincott Williams and Wilkins. USA. pp. 363-367.

Morrow, L.E., Gogineni, V. and Malesker, M.A. 2012. Probioics in the intensive care unit. Nutr. Clin. Prac. 27, 235-241.

Oskar, A., Meydani, S.N. and Russell, R. M. 2004. Yogurt and gut function. Am. J. Clin. Nutr. 80, 245-256.

Prado, F.C., Parada, J.L., Pandey, A. and Soccol, C.R. 2008. Trends in non-dairy probiotic beverages. Food Res. Int. 41, 111-123. 
Pundir, R.K., Rana, R., Kashyap, N. and Kaur, A. 2013. Probiotic potential of lactic acid bacteria isolated from food samples: an in vitro study. J. Appl. Pharm. Sci. 3, 85-93.

Pyar, H. and Peh, K.K. 2014. Characterization and identification of Lactobacillus acidophilus using biolog rapid identification system. Int. J. Pharm. Pharm. Sci. 6, 189-193.

Rao, K.P., Chennappa, G., Suraj, U., Nagaraja, H., Raj, A.P.C. and Sreenivasa, M.Y. 2015. Probiotic potential of Lactobacillus strains isolated from Sorghum-based traditional fermented food. Probiotics and Antimicro. Prot. 7, 146-156.

Saarela, M. 2002. Gut bacteria and health foods-the European perspective. Int. J. Food Microbiol. 78, 99117.

Sadrani, H., Dave, J. and Vyas, B.J.M. 2014. Screening of potential probiotic Lactobacillus strains isolated from fermented foods, fruits and of human origin. Asian $J$. Pharm. Clin. Res. 7, 216-225.

Salminen, S., Isolauri, E. and Salminen, E. 1996. Clinical uses of probiotics for stabilising the gut mucosal barrier: successful strains and future challenges. Antonie Van Leuwenhock. 70, 251-262.

Salvetti, E., Torriani, S. and Felis, G.E. 2012. The genus Lactobacillus: a taxonomic update. Probiotics \& Antimicro. Prot. 4, 217-226. DOI 10.1007/s12602-0129117-8
Schrezenmeir, J. and de Vrese, M. 2001. Probiotics, prebiotics, and synbiotics- approaching a definition. Am. J. Clin. Nutr. 73, 361S-364S.

Shahnaz, P., Shimazaki, K. and Kato, I. 2004. Milk and milk products in Bangladesh. J. Rakuno Gakuen Univ. 29, 916.

Smibert, R. M. and Krieg, N. R. 1994. Phenotypic characterization. In Methods for General and Molecular Bacteriology, 607-654. Edited by P. Gerhardt. Washington, DC: American Society for Microbiology.

Soccol, C.R., Vandenberghe, L.P.S., Spier, M.R., Medeiros, A.B.P., Yamaguishi, C.T., Lindner, J.D., Pandey, A. and Thomaz-Soccol, V. 2010. The potential of probiotics: A review. Food Technol. Biotechnol. 48, 413-434.

Suskovic, J., Brkic, B., Matosic, S. and Maric, V. 1997. Lactobacillus acidophilus M92 as potential probiotic strain. Milchwissenschaft. 52, 430-435.

Tambekar, D. H. and Bhutada, S. A. 2010. Studies on antimicrobial activity and characteristics of bacteriocins produced by Lactobacillus strains isolated from milk of domestic animals. The Internet J. Microbiol. 8, 1-6.

Tserovska, L., Stefanova, S. and Yordanova, T. 2000-2002. Identification of lactic acid bacteria isolated from katyk, goat's milk and cheese. J. Cult. Collect. 3, 48-52. 